\title{
A 50-year-old man with desmoid-type filbromatosis of upper extremity
}

\author{
Vitorino Modesto dos Santos, ${ }^{1,2}$ Viviane Vieira Passini Soares, ${ }^{1}$ Mayza Lemes Duarte, ${ }^{1}$ Priscilla Souza de Faria, ${ }^{1}$ \\ Uadson Silva Barreto, ${ }^{1}$ Victor Manabu Yano ${ }^{1}$ \\ ${ }^{1}$ Internal Medicine Department of Armed Forces Hospital, Brasília-DF; 2 ${ }^{2}$ atholic University Medical Course, Brasília-DF, Brazil
}

\begin{abstract}
This case study describes a 50-year-old man with a desmoid tumor of upper extremity, which had to be resected concomitant with the left arm amputation at middle third of the humerus. The course was initially stable, but evolved with aggressive infiltration of local soft tissues. During the watchful waiting time, three biopsies were done before establishing the diagnosis. Plain $\mathrm{X}$-ray, magnetic resonance and positron emission tomography were the exams utilized. The authors emphasize diagnostic and management challenges involving this rare condition. Case reports can stimulate further studies to get additional knowledge about this rare entity.
\end{abstract}

\section{Introduction}

Desmoid-type fibromatosis or desmoid tumor (DT) is an uncommon condition characterized by the absence of metastasis and frequent recurrences; ${ }^{1-7}$ it represents $3 \%$ of soft tissue tumors, and affects up to 0.4 per 100,000 of population, ${ }^{1,6}$ mainly between 6 to 40 years. ${ }^{5,7}$ DT is constituted by myofibroblasts with normal mitosis and variable local invasion; ${ }^{5-7}$ it may be progressive with recurrences or stable with spontaneous regression; watchful waiting approach is indicated in DTs of limbs or girdles. ${ }^{1-7}$ Radiotherapy, high-intensity focused ultrasound (MRgFUS), and surgery are therapeutic tools..$^{1-8}$

Correspondence: Vitorino Modesto dos Santos, Armed Forces Hospital, Estrada do Contorno do Bosque s/n, Cruzeiro Novo, 70658-900, Brasília-DF, Brazil.

Tel.: +55.61.39662103 - Fax: +55.61.32331599.

E-mail: vitorinomodestos@gmail.com

Key words: Aggressive fibromatosis; desmoid tumor; surgery; upper limb.

Conflict of interest: the authors declare no potential conflict of interest.

Received for publication: 12 March 2017.

Revision received: 27 July 2017.

Accepted for publication: 25 September 2017.

This work is licensed under a Creative Commons Attribution NonCommercial 4.0 License (CC BY-NC 4.0).

CC Copyright V. Modesto dos Santos et al., 2017

Licensee PAGEPress, Italy

Italian Journal of Medicine 2017; 11:403-406

doi:10.4081/itjm.2017.859

\section{Case Report}

A 50-year-old man was admitted in 2015 with swelling in the left shoulder and forearm (Figure 1) and local pain with evolution of five years. He denied fever or weight loss, and was tobacco smoker and alcohol abuser for 30 years. In 2011, local examination showed a mass adhered to deeper planes, and biopsy studies were unspecific. The positron emission chest tomography (PET-CT) showed hypermetabolic lesion $(8.7 \mathrm{~cm}$, standardized uptake values: 3.9$)$ in the left supra and infraclavicular regions (Figure 1B). Repeated biopsy revealed fibro adipose tissue without atypia. Immunohistochemistry showed mature adipose tissue and positive antibodies: CD3, CD20 and S100; whereas BCL2, AE1AE3, CD34 and CD31 were negative. Magnetic resonance (MRI) on admission (Figure 1C) showed a low signal heterogeneous mass $(12 \times 11 \times 8 \mathrm{~cm})$ with heterogeneous enhancement, compression of vasculo-nervous bundle, and involving the subscapular muscle. PET-CT showed increased mass and uptake compared with previous images (Figure $2 \mathrm{~A}$ and $\mathrm{B}$ ). Routine laboratory tests and tumor markers CA 19.9, CA 125, CEA and CA15.3 were unremarkable in 2013 and 2015. A third biopsy in 2015 showed fusocellular proliferation indicative of a low-grade mesenchymal tumor. Immunohistochemistry revealed a myofibroblastic lesion with the markers KI67 and AML positive; whereas EMA, S100, AE1AE3, CD34, desmin, and MUC-4 were negative. The surgery was postponed due to lung thromboembolism and lower limb deep venous thrombosis. Further control by MRI showed increased mass (Figure 2C and D), and the biopsy detected a fusocellular proliferation of low nuclear grade and infiltrative growth with characteristics of aggressive fibromatosis (Figure $3 \mathrm{C}$ and $\mathrm{D}$ ). The surgery occurred in June 2016, and showed infiltrated brachial plexus, and pericardium, pleura, and medi- 

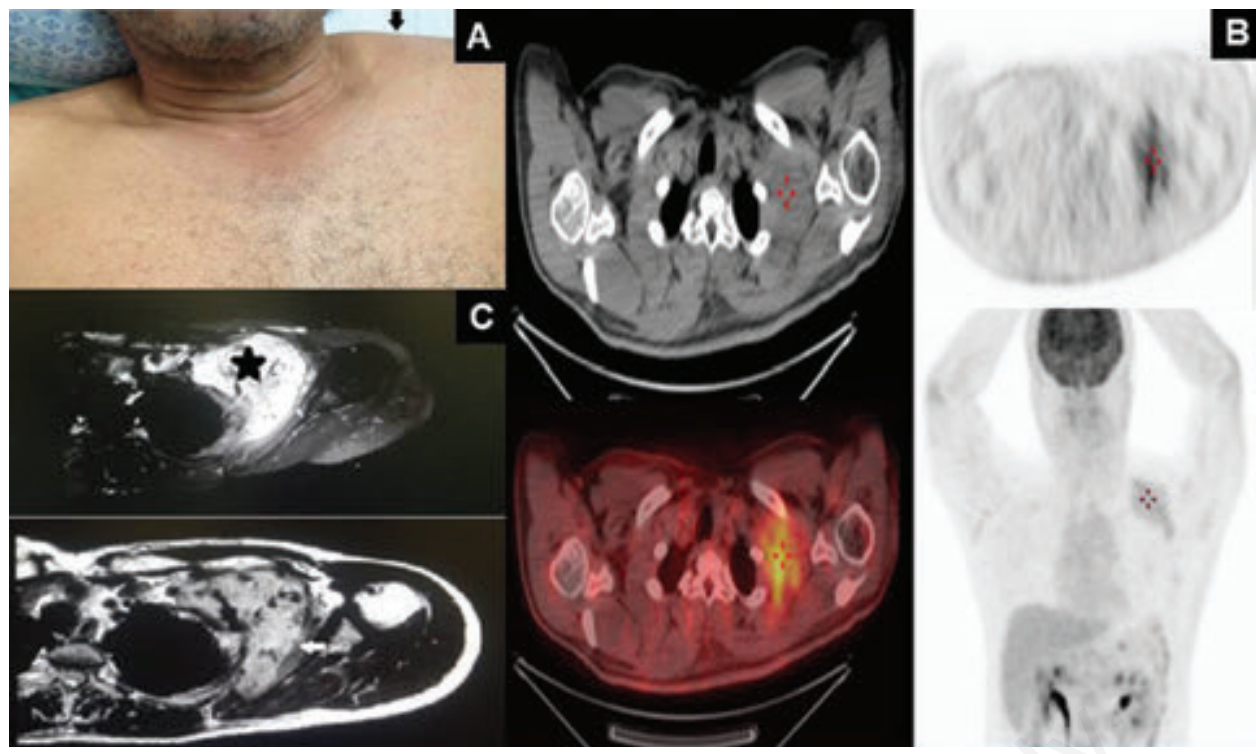

Figure 1. A) Gross evidence of the lump in left supraclavicular region (black arrow); B) Positron emission chest tomography in July 2013: images of the thorax showing a slightly hypermetabolic mass (red marker) in the left supra and infraclavicular fossa; and C) Magnetic resonance imaging of left shoulder in August 2015: bulky heterogeneous mass (arrow-T2 and starT1) with predominance of hypersignal, lobulated contours, poorly defined limits in the left lateral thoracic wall, extending from the supraclavicular region to subscapular area; possible origins were vascular, neural or sarcoma.

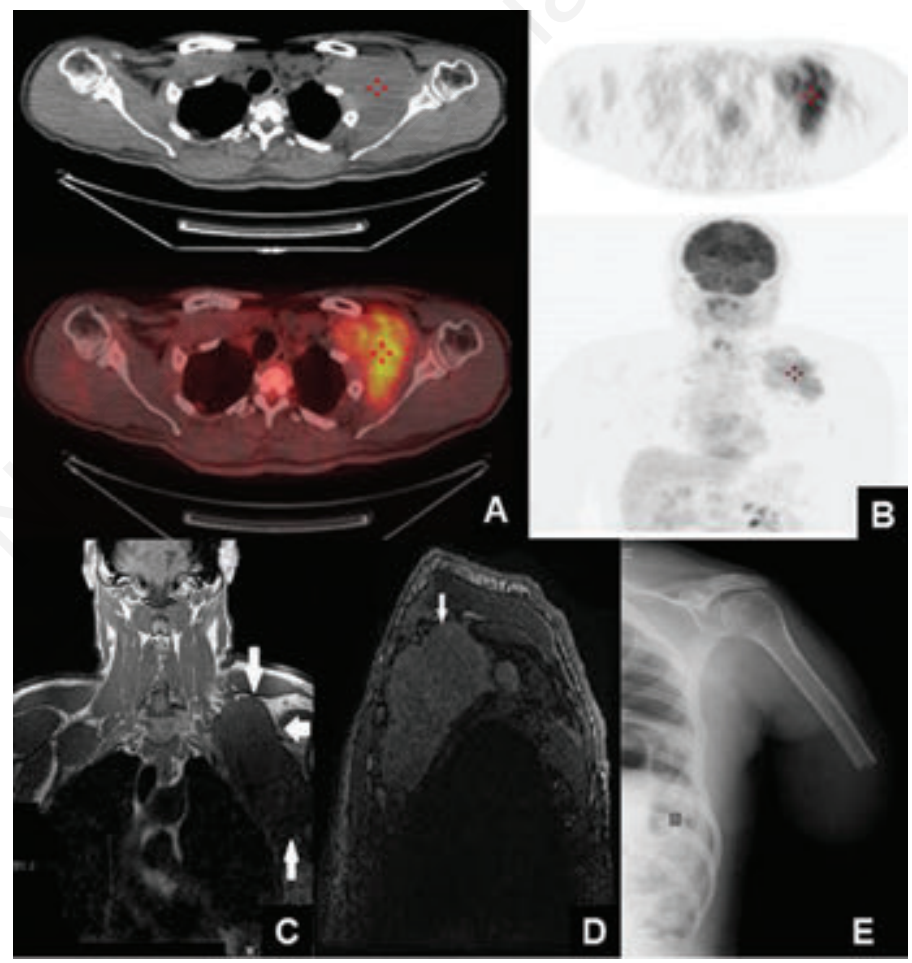

Figure 2. A and B) Positron emission chest tomography in August 2015: images of the hypermetabolic mass (red marker) with attenuation of soft parts mainly left infraclavicular, with supraclavicular and subscapular extension, with $11 \mathrm{~cm}$ in the largest axis [standardized uptake values (SUV): 4.5]. Slightly hypermetabolic retroperitoneal and left axillary lymph nodes up to $14 \mathrm{~mm}$ in small diameter (SUV: 1.8); C and D) Images of the extensive and heterogeneous mass (white arrow), with imprecise limits in the supra and left infraclavicular fossa, with predominant low signal in T1 and heterogeneous intermediate signal in T2; and E) Plain radiography in June 2016: Postoperative control image of the amputation performed at the mid-third level of the left humerus. 
astinum involvement. Limb amputation (Figure 2E) was the option. The patient is under thoracic surgery follow-up.

\section{Discussion}

DT of upper extremity herein described evolved six years with local pain and swelling. Diagnostic pitfalls were non-specific initial histopathologic data, and biopsy confirmed DT. ${ }^{5}$ Because of difficult management of DTs, the best surgical procedure is not consensual $;{ }^{1-7}$ the aggressive invasiveness of soft tissues led to the left upper limb amputation in this case. ${ }^{9}$ Houdek et al. studied 52 DTs of upper limbs followed by more than two years with $57 \%$ of 5 -year diseasefree. Radiotherapy increased the period of recurrence, but not disease-free time. ${ }^{2}$ Machado et al. reviewed predictive factors of recurrences and treatment in 51 cases of fibromatosis with seven years of mean follow-up. ${ }^{3}$ DTs of limbs or deep sited were more aggressive and recurrences did not depend on free margin or non-invasive control. ${ }^{3}$ Prudinger et al. reviewed prognostic factors for recurrence-free survival after surgery for DT of extremity in 27 patients with mean followup of 64 months. ${ }^{9}$ Fourteen had adjuvant radiotherapy,

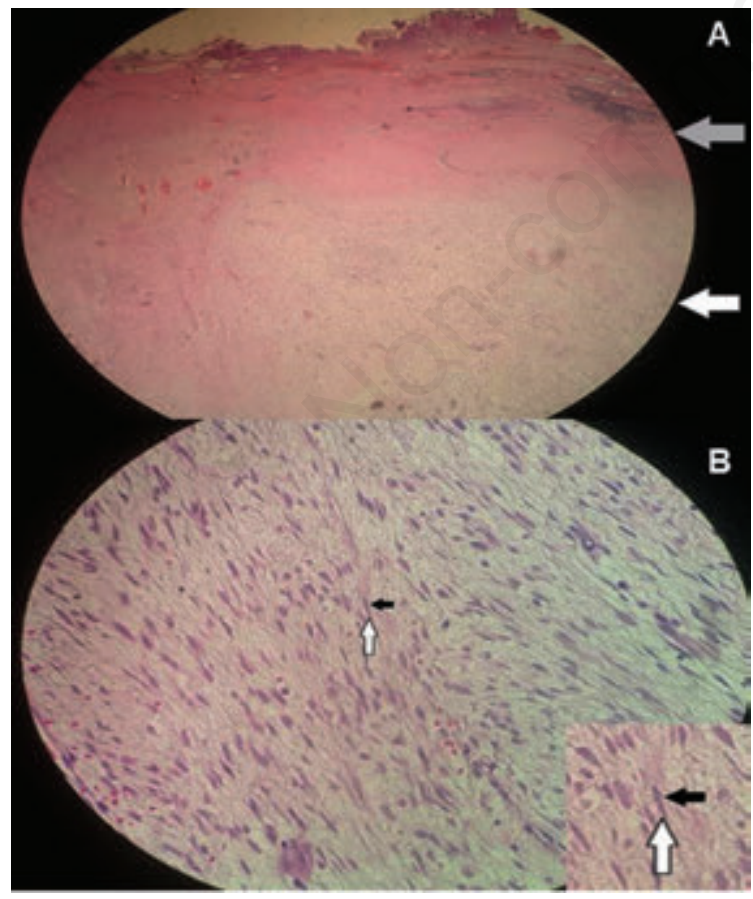

Figure 3. A) Photomicrograph of biopsy specimen showing the margin of normal tissue (dark arrow) next to the tumor (white arrow) limits [hematoxylin \& eosin (H\&E), $4 \times$; B) Details of the fusocellular proliferation, showing the features of the nucleus (black arrow) and the cytoplasm (white arrow) of the fibroblast (H\&E, 40×). which reduced the recurrences of the extremity tumors. ${ }^{9}$ Peng et al. evaluated clinicopathologic data and treatment of 211 cases of DT to identify factors of recurrences. ${ }^{6}$ The median size of tumors was $7.5 \mathrm{~cm}$, in limbs (32\%), abdomen, and thorax. Surgical margins were free in $60 \%$ of cases, the 5 -year recurrence-free survival was $52.8 \%$, and adverse factors were younger age and extra-abdominal site. ${ }^{6}$ Postoperative recurrences in 5 years occurred in $60 \%$ of cases. ${ }^{6}$ Santti et al. evaluated 41 patients with DT treated by radiotherapy and the significant response was $55 \% .^{7}$ The progression had no relationship with previous surgery or surgical margin. ${ }^{7}$ Tang et al. reviewed 21 patients with surgical resections of DTs with mean size of $10.5 \mathrm{~cm}$ aiming to preserve vital structures of shoulder girdle. The recurrence rate was $9.5 \%$, the mean follow-up was 49.8 months, and $14.3 \%$ of complications. ${ }^{10}$ Ver Halen reviewed 67 resections of DT with median tumor size of $9.8 \mathrm{~cm}$ in extremities (40\%), thorax, head and neck, and trunk. ${ }^{11}$ The 5-year recurrence-free survival was $73 \%$ for chest, abdomen and upper limb, and resection was not at expense of function preservation. ${ }^{11}$ Avedian et al. evaluated MRgFUS for more than 12 months in five cases of failures of treatment of DTs in popliteal fossa, buttock, posterior ankle and chest wall, and there was $36 \%$ of decrease in tumor size. ${ }^{8}$ Prudinger et al. reviewed recurrence-free survival after surgery of extremity DTs in 27 cases followed by 64 months, and radiotherapy improved 14 of them. ${ }^{9}$

\section{Conclusions}

The etiopathology and phenomena involved in recurrences of DT remain unclear; so, authors believe that case reports can stimulate prospective studies to get additional knowledge.

\section{References}

1. Gronchi A, Colombo C, Raut CP. Surgical management of localized soft tissue tumors. Cancer 2014;120: 2638-48.

2. Houdek MT, Rose PS, Kakar S. Desmoid tumors of the upper extremity. J Hand Surg Am 2014;39:1761-5.

3. Machado V, Troncoso S, Mejías L, et al. Risk factors for local recurrence of fibromatosis. Rev Esp Cir Ortop Traumatol 2017 [Epub ahead of print].

4. Otero S, Moskovic EC, Strauss DC, et al. Desmoid-type fibromatosis. Clin Radiol 2015;70:1038-45.

5. Pan F, Liu Q, Zhang G, et al. Aggressive fibromatosis of the leg and sacrococcygeal region: a report of two cases. Int J Clin Exp Pathol 2015;8:948-53.

6. Peng PD, Hyder O, Mavros MN, et al. Management and recurrence patterns of desmoids tumors: a multi-institutional analysis of 211 patients. Ann Surg Oncol 2012; 19:4036-42. 
7. Santti K, Beule A, Tuomikoski L, et al. Radiotherapy in desmoid tumors: treatment response, local control, and analysis of local failures. Strahlenther Onkol 2017 [Epub ahead of print].

8. Avedian RS, Bitton R, Gold G, et al. Is MR-guided highintensity focused ultrasound a feasible treatment modality for desmoid tumors? Clin Orthop Relat Res 2016;474:697-704.

9. Prodinger PM, Rechl H, Keller M, et al. Surgical resec- tion and radiation therapy of desmoid tumours of the extremities: results of a supra-regional tumour centre. Int Orthop 2013;37:1987-93.

10. Tang F, Min L, Yin R, et al. Large desmoid-type fibromatosis of the shoulder girdle: operative approach selection and clinic outcome. Int Orthop 2015;39:363-9.

11. Ver Halen JP, Soto-Miranda MA, Sandoval JA. Reconstruction of desmoid tumors: case series and systematic review. Ann Plast Surg 2015;75:480-6. 